\title{
THE EVOLUTION OF THE PLANETARY NEBULAE IN THE MAGELLANIC CLOUDS AND THE GALACTIC BULGE
}

\author{
M.A. DOPITA \\ Mt. Stromlo and Siding Spring Observatories, Institute of Advanced Studies, \\ The Australian National University
}

\begin{abstract}
From self-consistent photoionisation modelling of 147 Magellanic Cloud PN, we have constructed the H-R Diagram for the central stars, and have derived both the chemical abundances and the nebular parameters. We find that the central stars have core masses generally between 0.55 and $0.7 \mathrm{M}_{\odot}$, and find strong evidence to support the model that they leave the AGB as helium-burning stars following the final shell flash. Type I PN have more massive cores, up to near the Schwartzschild limit, and show clear evidence for the Third dredge-up episode. From HST images, younger PN are very compact. The expansion velocity of the nebula is closely correlated with the position of the central star on the H-R Diagram.
\end{abstract}

\section{Introduction}

The study of the nearby Galactic Bulge and Magellanic Clouds (MC) populations of planetary nebulae provides a vital key to our understanding of the details of post-asymptotic Giant Branch evolution. These PN are near enough to be studied in detail, are at known distance, and details of their internal structure can be resolved. A comparison of the relatively young, metal poor MC population with the PN in the Galactic Bulge would be expected to allow the evolutionary effects of both age and metallicity of the parent stars to be distinguished and understood.

The MC population has been the subject of a systematic and detailed study by us and by the University College group in recent years, and by the time of IAU Symposium \#131, data on the diameters, fluxes, expansion velocities and kinematics had all been accumulated (see the review by Barlow, 1989, also Monk, Barlow and Clegg 1988,Dopita et al. 1985; Dopita, Ford, and Webster 1985; Meatheringham et al. , 1988 ; Meatheringham, Dopita, Morgan 1988 ; Wood, Bessell, and Dopita, 1986; Wood et (al., 1987). This has led to a general understanding of the outlines of an evolutionary sequence (Dopita and Meatheringham, 1990), a necessary prerequisite to detailed modelling of individual PN. Since then, the acquisition of high quality spectrophotometry for some 147 PN in the Magellanic Clouds has permitted rapid progress to be made in the detailed modelling of these PN and their central stars.

Likewise the Galactic Bulge population has been subjected to intense study in recent years. A number of new objects have been discovered by optical techniques (Kinman, Feast and Lasker 1988). However, the most productive technique has been to identify PN candidates by their IRAS colours, and then to subsequently search for the radio continuum emission from these objects (Pottasch et al. 1988; Pottasch, Ratag and Olling 1990; Ratag et al.1990; Ratag 1990). Absolute H $\beta$ fluxes have been measured for many PN by Acker et al. 1991). Extensive Spectrophotometric data of high quality has been obtained by Webster (1988), Ratag (1990) and Acker et al. (1991). This raises the number of objects observed to almost 300, permitting detailed comparison of the Bulge and the Disk populations of PN. 


\section{Dynamical and Size Evolution.}

For the MC PN, dynamical evidence (Dopita et al. 1987) leads us to the adoption of a two-wind model (Kwok et. al. 1978; Kwok 1982) to describe the outlines of the evolutionary sequence for PN. In this model, the ionised gas of the PN will be trapped between the compressed AGB wind and a hot pad of shocked stellar wind. In the particular case that the total energy content can be represented by a simple power law in time, $E(t)=E_{o} t^{\alpha}$, and if the radial density distribution in the undisturbed AGB wind is given by a power law in radius, $\rho(r)=\rho_{o} r^{\beta}$, then, from dimensional considerations, the radius of the outer shock $R$, and the velocity of expansion $V_{\text {exp }}$, are given by:

$$
R=A .\left(E_{o} / \rho_{o}\right)^{1 /(5+\beta)} \cdot t^{(2+\alpha) /(5+\beta)} ; \quad V_{\text {exp }}=B .\left(E_{o} / \rho_{o}\right)^{1 /(5+\beta)} \cdot t^{(\alpha-\beta-3) /(5+\beta)}
$$

A and B being dimensionless constants. If we assume that the nebula is evolving into a AGB wind that has been blown at a steady mass-loss rate and velocity $(\beta=-2)$, then the observational material on the Magellanic Cloud PN limits $\alpha$ to lie in the bounds $1<\alpha<2$. This result is in accord with radiative-driven wind theory since the PNn evolutionary models of Wood and Faulkner (1986) predict $\alpha$ to lie in the theoretical range $1<\alpha<1.7$. In the limiting cases, $\alpha=1$ and $\alpha=2$, we have, for $\beta=2$ :

$$
\begin{array}{lll}
\alpha=1.0 ; & V_{\text {exp }}=\text { const. } ; & R=\text { const. } t \\
\alpha=2.0 ; & V_{\text {exp }}=\text { const. } R^{1 / 4} ; & R=\text { const. } t^{4 / 3}
\end{array}
$$

The velocity of expansion therefore depends only very weakly on radius during the optically thick evolution, a result supported by observations (Sabbabin and Hamzaoglu, 1982; Phillips, 1984; Hippelein and Weinberger 1990). The weakness of this dependence makes it a poor test of theoretical evolutionary scenarios, despite its fairly extensive use in the literature (e.g.Sabbadin $e t$ al., 1984; Okorokov et al., 1985).

From the MC sample, we find that the effective temperature, luminosity, and the velocity of expansion of the nebula are well correlated through

$$
\left(V_{\text {exp }} / \mathrm{km} \cdot \mathrm{s}^{-1}\right)=-128 \pm 4+38 \pm 2\left[\log \left(T_{\text {eff }}\right)-0.25 \pm 0.05 \log \left(L / L_{\odot}\right)\right]
$$

It is clear that this relationship is fundamental to the understanding of the dynamical evolution of PN, showing that the PN shells are accelerated continuously during the evolution of the PNN towards the blue. This acceleration occurs early for low-mass PN, but higher terminal velocites are reached in the case of the high-mass PN. This is consistent with a lower shell mass in the low mass PN, and more energetic stellar winds in the high-mass PN.

In this model, the stellar wind provides the pressure which confines the ionised material to a (somewhat) thin shell. Gathier et al. (1983), and, more recently, Pottasch and Acker (1989) and Dopita and Meatheringham (1990) have pointed out that the strong relationship between nebular mass and nebular radius is strong evidence to support the idea that these PN are optically thick. However, for the Galactic Bulge PN, both Zijlstra (1990) and Stasinska et al. (1991) have argued that this relationship may simply due to selection effects, or artefacts due to error propagation, and that most PN are density bounded. However, this conclusion seems to be at variance with spectral data, for which the relative strength of the low ionisation lines appears to exclude the possibility that the nebulae are optically thick in the majority of cases.

For the Galactic Bulge PN, there appears to be room for improvement in the measurement of their diameters. For PN smaller than about 5 arc sec. in diameter, the radio continuum and optical 
diameters do not agree, in the sense that the optical diameters are larger. However, where carefully calibrated optical observations have been made (Dopita et al. 1990), the optical diameters agree very well with the radio sizes. It appears therefore that optical sizes for Galactic Bulge PN should be treated with caution, and conclusions concerning the mass-radius relation are premature.

In the last year, HST images have been obtained for a number of PN in the Magellanic Clouds (see Dopita et al., this conference). This data clearly shows that the smallest PN are more compact than this expected, so that their dynamical ages are smaller than would be expected from theory. This implies that the final phase of mass ejection was denser, and occurred at a lower velocity $\left(\sim 3 \mathrm{~km} . \mathrm{s}^{-1}\right)$ than the simple AGB wind model would predict. It is tempting to associate this mass ejection with a "superwind" generated during the final helium shell flash. If this were the case, PN would leave the AGB as helium-burners (see below). For these objects, the dynamical age is simply the time taken for the newly-ionised gas to flow out of the central region. For optically thick PN, simple Strömgren theory predicts that the ionised mass $M_{\text {neb }} \propto\left(L_{*} R^{3}\right)^{1 / 2}$. Hence, we may define a reduced mass which is the nebular mass corrected to unit luminosity of the central star. This is shown in Figure 1. The slope defined by these points is close to unity, rather than the value of $3 / 2$ that would obtain for constant filling factor. The implication is that the total thickness of the ionised shell remains approximately constant during the expansion of the nebula, i.e. the filling factor of the ionised shell varies as $R^{-1}$. This is clear evidence that the PNn wind has a major rôle in shaping and accelerating the nebular shell in the later phases of evolution (see Marten and Schönberner 1991).

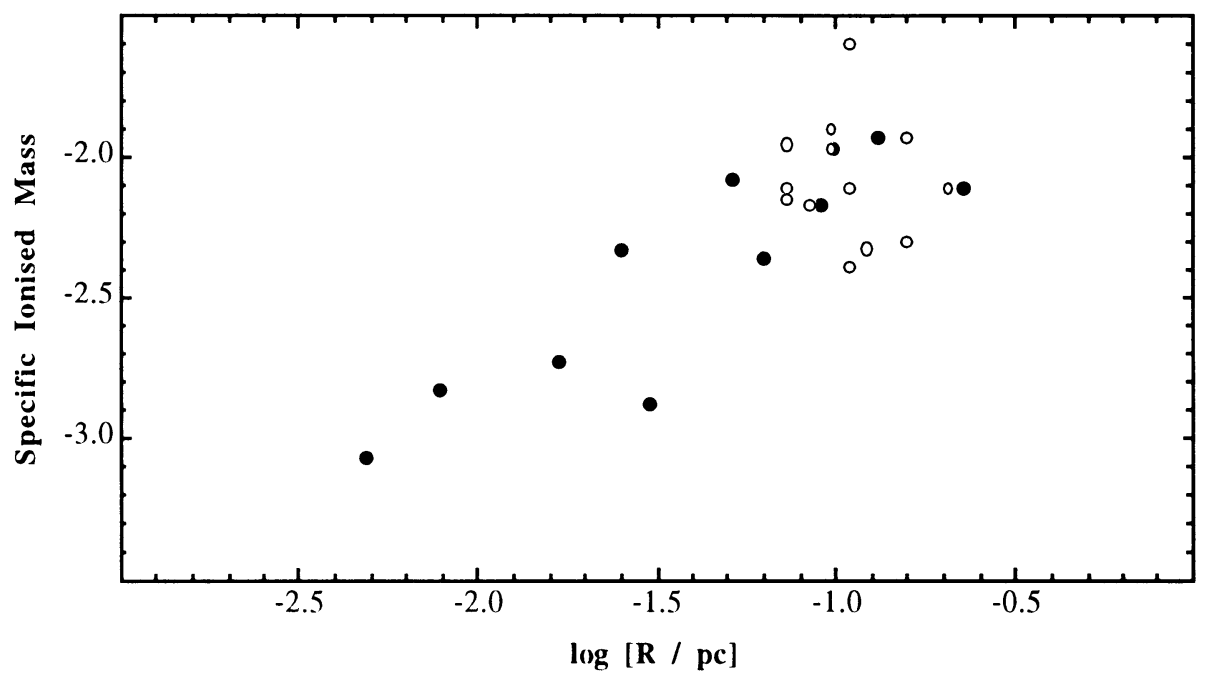

Figure 1: The reduced mass: radius relation for the LMC PN for which the [O III] sizes have been directly measured. The filled circles are from HST PC images (see Dopita et al., this volume), and the open circles are from the imaging of Jacoby, Walker and Ciardullo (1990).

\section{The Observed Hertzsprung-Russell Diagram.}

For the Magellanic Clouds we use the spectrophotometric results presented by Meatheringham and Dopita (1991a,b) and of Vassiliadis et al. (1992). The objects are drawn from the Sanduleak, MacConnell and Philip (1978) list; a fairly uniform magnitude limited sample. Fainter PN such as 
the Jacoby objects studied by Henry, Leibert and Boronson (1989), are not well represented. However, recently a number of new planetary nebulae (PN) have been found the outer fields of the LMC ( Morgan and Good, 1992; Morgan 1992). Many of these objects have been confirmed as PN and spectrophotometry has been obtained (Dopita, Vassiliadis and Morgan, this volume).

In order to determine the PN nebular abundances, and the position of the central star on the $\mathrm{H}-\mathrm{R}$ diagram we require to know the absolute $\mathrm{H} \beta$ flux, the nebular density, and to have accurate spectrophotometry over as wide a wavelength as possible. This is possible because, with the aid of a photoionisation code, the ionisation temperature can be determined from the nebular excitation, the luminosity of the central star can be determined from the absolute $\mathrm{H} \beta$ flux, and the chemical abundances can be determined from the electron temperature of the nebula and from its detailed emission line spectrum. We have used the generalised modelling code MAPPINGS (Binette, Dopita and Tuohy, 1985) and its major upgrade MAPPINGS 2 (Sutherland and Dopita 1992) to compute the emission line spectra of isobaric nebulae in photoionisation equilibrium with central stars having a Black-Body photon distribution.

The "observed plane" of the H-R Diagram for the Magellanic Cloud PN is the Excitation Class - H $\beta$ Flux plane, since Excitation Class is closely related to $T_{\text {eff }}$, and $\mathrm{H} \beta$ Flux is closely related to $L_{*}$. The challenge for the modeller is to discover the transformation.

The detailed definition of excitation class, $E$, differs somewhat from author to author (c.f. Aller 1956; Feast 1968; Webster 1975; and Morgan 1984). We will use here the classification given by Dopita and Meatheringham (1990), which, since it was defined in terms of two line ratios, is a continuous variable. Our modelling allows us to derive an excitation temperature, related fundamentally to these two line-ratios, but involving many other diagnostic line ratios. Since our models are self- consistent, abundance effects are largely eliminated. This method is closely related to the so-called energy balance method developed by Preite-Martinez and Pottasch (1983) from an original idea of Stoy (1933). In general, a temperature derived by a global model which allows for abundance variations should be more accurate. From our models, at MC abundances, we find that the correlation between E.C. and $\log \left(T_{\mathrm{eff}}\right)$ is:

$$
\log \left[T_{\mathrm{eff}}\right]=4.489+0.112 . E-0.0017 . E^{2}
$$

Given the effective temperature, the luminosity of the central star is determined by the absolute $\mathrm{H} \beta$ flux. For the case of optically thick objects, our models show that reddening corrected $\mathrm{H} \beta$ flux can be represented as a function of luminosity and excitation class:

$$
\log \left[L_{*} / L_{\odot}\right]=\log \left[L_{H \beta}\right]-31.262-0.179 E+0.035 E^{2}-0.00166 E^{3}
$$

This relationship is only weakly metallicity dependent (Dopita, Jacoby and Vassiliadis 1992). Optically-thin PN may also be placed on the H-R diagram, though with lower accuracy. The effective temperatures and luminosities of the central stars, as derived from the detailed photoionisation modelling of only the optically thick PN, are given in Figure 2. A distance modulus to the LMC of 18.5 (corresponding to a distance of $50 \mathrm{kpc}$ ) has been assumed. Figure 2 multiplies the sample of Magellanic Cloud PNn which have been placed on the H-R Diagram by a factor of five, previous studies being those of Aller et al. (1987), and Monk, Barlow and Clegg (1988). The bright optically thick objects are found in a range of core masses between $0.56-0.70 \mathrm{M}_{\odot}$, with a mean of about $0.62 \mathrm{M}_{\odot}$. This is consistent with the results of Barlow (1989), or Dopita and Meatheringham (1990). However, it is very evident that the mean mass is higher, and the range of core masses wider, than those derived by Schönbemer (1981) for a group of nearby, evolved PNn. Note that Type I PN are found preferentially at high $\mathrm{T}_{\text {eff }}$, lower luminosities and, in general, at larger values of the core mass $\left(\mathrm{M}_{\text {core }} \geq 0.7 \mathrm{M}_{\odot}\right)$. This is consistent with the assumption that these PN represent the more massive progenitor stars. Optically-thin PN occupy a broader region of the 
diagram, of somewhat lower mean mass, from 0.546 to $0.65 \mathrm{M}_{\odot}$, implying that the efficiency of mass loss during the AGB is a very strongly decreasing function of the core mass and luminosity.
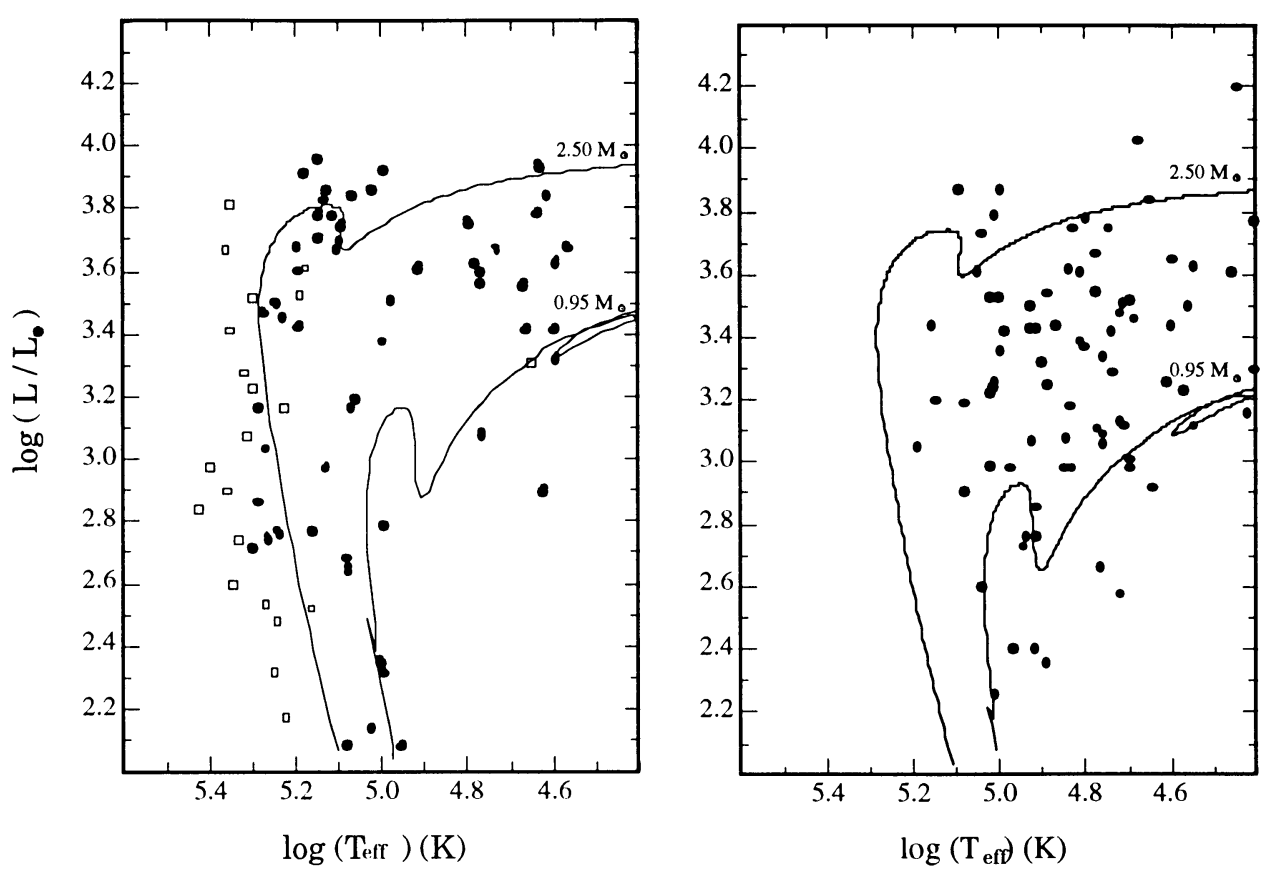

Figure 2: The observed H-R Diagram for the optically-thick PN in the LMC (left), and the corresponding diagram for the Galactic Bulge PN, taken from Ratag (1991), compared with theoretical tracks for Heburning stars from Vassiliadis and Wood (1992). These correspond to core masses of 0.57 and $0.68 \mathrm{M}_{\odot}$. These tracks appear to be able to better describe the observed distribution of PN. In particular, the Galactic Bulge PN in the region $\log \mathrm{L}<3.0, \log \mathrm{T}_{\mathrm{eff}}<5.0$ would be difficult to account for in the case of hydrogen-burning models. It is clear that the differences between these two H-R Diagrams are mainly due the the different ages of the two populations. The LMC Type I PN are shown as open squares, and clearly represent a high-mass population, regardless of the burning status of the central star.

The positions of the Galactic Bulge PN on the H-R Diagram has been best determined by Ratag (1991) using the energy-balance technique, fully accounting for energy losses in the IR. The agreement he finds between this technique and the measured Zanstra temperatures gives credence to the results, and lends further support that most of the optically-observed PN are optically thick to the ionising radiation. These results are also shown in Figure 2. Note the absence of luminous, high-temperature stars, corresponding to young, massive PNN. Also, the relatively large numbers of objects in the region $\log \mathrm{L} / \mathrm{L}_{\odot}<3.0 ; \log \mathrm{T}_{\text {eff }}<5.0$. Both of these results are consistent with the older PN population in the Bulge.

\section{Comparision of the Observed and the Theoretical Hertzsprung-Russell Diagrams}

The availability of evolutionary tracks for the PNn has been somewhat limited (Paczynski 1971; Harm and Schwartzschild 1975; Schönberner 1981; Iben 1984; Wood and Faulkner 1986). 
Recently however, Vassiliadis and Wood (1992) presented fully self-consistent tracks fom the Main Sequence though to the White Dwarf stage. This work demonstrated that the most important factor determining the relationship of the PNN to the main-sequence star from which it had evolved is the treatment of mass-loss on the AGB. In the Vassiliadis and Wood work, this was treated in an internally consistent manner which gives the observed period-luminosity relationship for the long period variables. For the first time, it is possible to relate the initial mass $M$ and metallicity $[\mathrm{Z}]$ of the star to the age of the star when it reaches the PN phase of evolution $\tau$, its core mass $M_{\text {core }}$, and its (hydrogen-burning ) luminosity (Dopita, Jacoby and Vassiliadis 1992). Empirically;

$$
\begin{aligned}
(\tau / \text { Gyr }) & =14.35[\mathrm{Z}]^{0.195}\left(M / M_{\odot}\right)^{-(3.4+\log [\mathrm{Z}])} \\
\left(M_{\text {core }} / M_{\odot}\right) & =0.493[\mathrm{Z}]^{-0.035}\left\{1+0.147\left(M / M_{\odot}\right)\right\} \\
\left(L / L_{\odot}\right) & =57340\left\{\left(M_{\text {core }} / M_{\odot}\right)-0.507\right\} .
\end{aligned}
$$

and

Usually, the observed H-R diagram for PNN is compared with hydrogen-burning tracks. However, the observed distribution of points in Figure 2 is difficult to reconcile with this hypothesis for a number of reasons. First, the rapidly accelating rates of evolution as we go to higher core masses, and the rapidly decreasing number of stars of higher mass resulting from the slope of the IMF means that the luminosity function PNN should be very rapidly decreasing above $\log \left(\mathrm{L} / \mathrm{L}_{\odot}\right) \sim 3.7$ (Shaw 1989). This is not seen. Second, the clump of LMC points in the vicinity of $4.0>\log \left(\mathrm{L} / \mathrm{L}_{\odot}\right)>3.7 ; 5.2>\log \left(\mathrm{T}_{\mathrm{eff}}\right)>5.05$ has no explanation. Hydrogen-burning models evolve through here in less than $1000 \mathrm{yr}$. Third, the Type I PN are highly unlikely to be seen in the region of the H-R diagram above $\log \left(\mathrm{L}_{\mathrm{L}} \mathrm{L}_{\odot}\right) \sim 2$, since their fading times are so rapid, if hydrogen burning. Finally, the existence of PN in the region $\log \left(\mathrm{L} / \mathrm{L}_{\odot}\right)<3.0 ; \log \left(\mathrm{T}_{\mathrm{eff}}\right)<5.0$ found by Ratag, Pottasch and Waters (1992) cannot be explained. These would have to have core masses in the range 0.54$0.58 \mathrm{M}_{\odot}$, and such objects would have ages greater than the Universe, according to the above equations.

All these results can be reconciled if the PNN eject a large amount of matter in the final shell flash event, causing them to leave the AGB as helium-burners. The PNN fade until hydrogen reignition takes place, causing the characteristic dog-leg in the tracks of Figure 2. At this time the rate of evolution across the H-R diagram is slowed, allowing a greater probability for objects to be observed in this phase. In the helium-burning tracks, the rate of evolution blueward is very much less mass-dependent that for the hydrogen-burning tracks, and the fading of more massive objects is considerably delayed, allowing the Type I objects to be observed while still relatively bright.

The distribution of the brighter PNN in Figure 2, if interpreted as helium-burners implies that the rate of star formation in the LMC has not been uniform over time. The faintest PNn are consistent with the lower track, for which the stellar age $~ 15$ Gyr. This is similar to the Bulge and to the older globular clusters in our Galaxy, suggesting that both the Galaxy and the LMC formed at about the same epoch. For $\log \left(\mathrm{T}_{\mathrm{eff}}\right)>5.0$, there is a concentration of points near the $1.5 \mathrm{M}_{\odot}$ track $\left(0.62 \mathrm{M}_{\odot}\right.$ core mass). This would imply a burst of star formation about 3-4 Gyr ago. This age estimate is similar to that arrived at by Meatheringham et al. (1988) from a study of the kinematics of the PN (2.5-3.6 Gyr), where it was assumed that the current day vertical velocity dispersion has been achieved by orbital diffusive processes operating over the lifetime of the precursor star. Finally, the large concentration of points near and above the $2.5 \mathrm{M}_{\odot}$ track implies that a burst of star formation took place within the last $\sim 3 \times 10^{8}$ yr. Both these major bursts coincide reasonably well with those estimated from the distribution of ages of open clusters (van den Bergh 1991), and are coincident with the major bursts identified in the field star Giants (Frogel and Blanco 1983; Stryker 1984 , and references therein). 


\section{The Evolutionary Status of the Type I PN in the Magellanic Clouds.}

It has been frequently assumed, on the basis of evolutionary models with dredge up, that the N-rich Type I PN are derived from a more massive population than ordinary PN. The position of the LMC Type I PN in Figure 2 clearly proves that this assumption is correct. In fact, it is likely that for a number of Type I PN, the luminosity is underestimated because UV radiation can escape (poleward) through the characteristic ansae which these PN display. Indeed, images taken with the HST (Dopita et al., this volume) confirm this to be the case in at least two Type I objects (SMP \#83 and 96). In the case of SMP\#83, the equatorial ring of nebular gas and the optically-thick lobes together intercept only $\sim 10 \%$ of the ionising radiation, meaning that the true luminosity of the central star is $\sim 4 \times 10^{4} \mathrm{~L}_{\odot}$. Likewise the nucleus of the Type I PN N67 (SMP\#22) in the SMC has been detected at X-ray wavelengths, placing it at $\log \left(\mathrm{L} / \mathrm{L}_{\odot}\right)=4.6 \pm 0.7 ; \log \left(\mathrm{T}_{\text {eff }}\right) \sim 5.5$ (Wang, $1991)$. These figures imply core masses of order $1.2 \mathrm{M}_{\odot}$ for both objects.

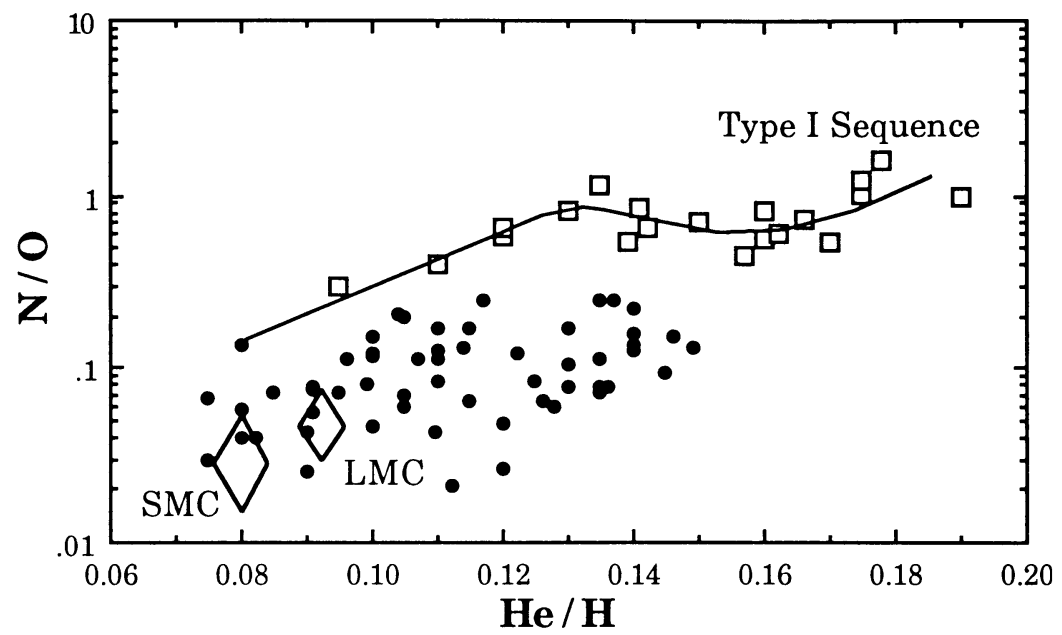

Figure 3: The N/O ratio as a function of $\mathrm{He} / \mathrm{H}$ ratio for the luminous optically-thick $\mathrm{PN}$ in both the

LMC and the SMC (from Dopita and Meatheringham, 1991b). The mean abundances in the ISM of each galaxy is shown as an error diamond from Russell and Dopita (1992). The Type I PN show as a distinct sequence on this diagram.

The abundance differences between the Type I objects and the sample as a whole is clearly exemplified in figure 3 , in which we plot the N/O ratio as a function of helium abundance. Many points are found near the mean points for the LMC and the SMC found by Russell and Dopita (1990), and Type I objects stand out as having both high He/H ratios and high N/O ratios. Such a diagram has been extensively used by Kaler $(1983 ; 1985)$ as a diagnostic of the importance of the various dredge-up processes occuring during the first Giant and AGB phase of evolution (Becker and Iben 1980; Renzini and Voli 1981; Iben and Renzini 1983). It is apparent from figure 3 that some objects show appreciable He enhancements without any corresponding $\mathrm{N} / \mathrm{O}$ enhancement; a signature of the third dredge up phase without $\mathrm{C} / \mathrm{N}$ conversion. Since the Type I objects have been shown to have more massive core masses, and hence more massive precursors, we can conclude that the $\mathrm{C}$ to $\mathrm{N}$ conversion is mass-dependent. Lastly, for a number of Type I PN, there appears to be a depletion in the absolute abundance of $O$, relative to other $\alpha$-process elements such as $\mathrm{Ne}$ (Aller et al. 1987; Dopita and Meatheringham 1991). This appears to be evidence for the dredge up of ON-processed material, which as yet has no clear theoretical explanation. 


\section{Conclusions}

Since the last IAU Symposium \#131, the study of the H-R Diagrams of PN has finally reached the stage where a detailed confrontation of observation and theory has become possible. In this review, we have shown how the dynamical / radius evolution is determined by AGB mass loss from the central star and by the high speed wind during the PN phase of evolution. An excellent agreement between the observed and the theoretical evolutionary tracks is obtained only provided that the PN leave the AGB as helium-burning objects following a shell flash. On this interpretation, details of the star formation history of the LMC may be distiguished, which agree with other techniques. The Type I PN, are found to have high core masses, and to lie on the descending branch of the evolutionary tracks. They show evidence for a 3 rd. dredge-up episode resulting in correlated He and $\mathrm{N}$ abundance enhancements. We also find clear evidence, most notably in the Type I objects for dredge-up and the ejection of ON processed material into the PN envelopes.

\section{References}

Acker, A., Stenholm, B., Tylenda, R., and Raytchev, B. 1991a, Ast. Ap., 90, 89.

Acker, A., Köppen, J., Stenholm, B., and Raytchev, B. 1991b, Ast. Ap. Suppl. Ser., 89, 237.

Aller, L.H. 1956 Gaseous Nebulae (New York:Wiley).

Aller, L.H., Keyes, C.D., Maran, S.P., Gull, T.R., Michalitsianos, A.G. and Stecher, T.P., 1987 Ap. J., 320, 159.

Barlow, M.J. 1987, M. N. R. A. S., 227, 161. 1989 in IAU Symp. \#131"Planetary Nebulae", Ed. S.Torres-Peimbert (Kluwer:Dordrecht), p319.

Becker, S.A., and Iben, I. Jr. 1980, Ap. J., 237, 111.

Binette, L., Dopita, M.A., and Tuohy, I.R. 1985, Ap. J., 297, 476.

Dopita, M.A., Ford, H.C., Lawrence, C.J., and Webster, B.L. 1985, Ap. J., 296, 390.

Dopita, M.A., Ford, H.C., and Webster, B.L. 1985, Ap. J., 297, 593.

Dopita, M.A., Henry, J.P, Tuohy, I.R., Webster, B.L., Roberts, E.H., Y-I. Byun, Cowie, L.L. and Songaila, A., 1990, Ap. J., 365, 640.

Dopita, M.A., Jacoby, G.H., and Vassiliadis, E. 1992, Ap. J., 389, 27.

Dopita, M.A., and Meatheringham, S.J. 1990 Ap. J., 357, 140. $1991 \mathrm{a}$, Ap. J., 367, 115. 1991b, Ap. J., 377, 480.

Dopita, M.A., Meatheringham, S.J., Webster, B.L., and Ford, H.C. 1988, Ap. J., 327, 639.

Dopita, M.A., Meatheringham, S.J., Wood, P.R., Webster, B.L., Morgan, D.H., and Ford, H.C.,1987, Ap. J. (Lett.), 315, L107.

Feast, M.W. 1968, M. N. R. A. S., 140, 345.

Frogel, J.A., and Blanco, V.C., 1983, Ap. J., 274, L57.

Gathier, R., Pottasch, S.R., Goss, W.M.,and van Gorkom, J.M. 1983, Ast. Ap., 128, 325.

Harm, R., and Schwartzschild, M., 1975, Ap.J., 200, 324.

Henry, R.B.C., Liebert, J., and Boroson, T.A., 1989, Ap. J., 339, 872.

Hippelein, H., and Weinberger, R. 1990, Ast. Ap., 232, 129.

Iben, I., Jr. 1984, Ap. J., 277, 333.

Iben, I., Jr., and Renzini, A. 1983, Ann. Rev. Astr. Ap., 21, 271.

Jacoby, G.H., Walker, A.R., and Ciardullo, R. 1990, Ap. J., 365, 471.

Kaler, J.B. 1983, "Planetary Nebulae", ed. D.R. Flower (Reidel:Dordrecht),p245. 
1985 Ann. Rev. Ast. Ap., 23, 89.

Kaler, J.B., and Jacoby, G.H. 1990, Ap. J., 362, 491.

Kinman, T.D., Feast, M.W., and Lasker, B.M.. 1988 AJ 95, 804.

Kwok, S. 1982, Ap. J., 258, 280.

Kwok, S., Purton, C.R., and FitzGerald, P.M. 1978, Ap. J. (Lett), 219, L125.

Martin, H., and Shönberner, D. 1991, Ast. Ap., 248, 590.

Meatheringham, S.J., and Dopita, M.A., 1991a, Ap. J. Suppl. Ser., 75, 407. ., 1991b, Ap. J. Suppl. Ser., 76, 1085.

Meatheringham, S.J., Dopita, M.A., Ford, H.C., and Webster, B.L. 1988, Ap. J., 327, 651.

Meatheringham, S.J., Dopita, M.A., and Morgan, D.H., 1988 Ap. J., 329, 166.

Monk, D.J, Barlow, M.J., and Clegg, R.E.S. 1988 M.N.R.A.S., 234, 583.

Morgan, D.H. 1984, M.N.R.A.S., 208, 633.

Morgan, D.H., andGood, A.R., 1985, Mon Not. R.A.S., 213, 491.

Morgan, D.H. 1992, (in prep).

Okorokov, V.A., Shustov, B.M., Tutukov, A.V., and Yorke, H.W., 1985, Ast. Ap., 142, 441.

Paczynski, B. 1971, Acta Astron., 21,417.

Phillips, J.P., 1984, Astr. Ap., 137, 92.

Pottasch, S.R., and Acker, A., 1989, Ast. Ap., 221, 123.

Pottasch, S.R., Bignell, C., Olling, R., and Zijlstra, A.A. 1988, Ast. Ap., 205, 248.

Pottasch, S.R., Ratag, M. A., and Olling, R. 1990, in "From Miras to PN - Which path for Stellar Evolution?", eds M.O. Mennessier and A. Omont, Editions Frontières, France, p381.

Preite-Martinez, A., and Pottasch, S.R., 1983, in IAU Symp\#103, "Planetary Nebulae", ed. D.R. Flower, (Reidel:Dordrecht), p547.

Ratag, M.A. 1990, Thesis, U.of Groningen.

Ratag, M.A., Pottasch, S.R., and Waters, L.B.F.M., 1992, Ast. Ap. (in press).

Ratag, M.A., Pottasch, S.R., Zijlstra, A.A., and Menzies, J. 1990, Ast. Ap., 233, 181.

Renzini, A. and Voli, M. 1981, Ast. Ap., 94, 175.

Sabbadin, F., Gratton, R.G., Bianchini, A., and Ortolani, S., 1984, Ast. Ap., 136, 181.

Sabbabin, F. and Hamzaoglu, E., 1982, Ast. Ap., 110, 105.

Sanduleak, N., MacConnell, D.J., and Philip, A.G.D., 1978, P. A. S. P., 90, 621.

Schönberner, D., 1981, Astr. Ap., 103, 119.

Shaw, R.A. 1989, in IAU Symp. \#131"Planetary Nebulae", Ed. S.Torres-Peimbert

(Kluwer:Dordrecht), p319.

Stasinska, G., Tylenda, R., Acker, A., and Stenholm, B. 1991, Ast. Ap., 247, 173.

Stoy, R.W., 1933, M.N.R.A.S., 93, 588.

Stryker, L.L., 1984, IAU Symp\#108, "Structure and Evolution of the Magellanic Clouds, eds. S. van den Bergh and K.S. de Boer, Dordrecht:reidel, p79.

Sutherland, R.E., and Dopita, M.A. 1992, Ap. J. Suppl., (in press).

Vassiliadis, E, Dopita, M.A., Morgan, D.H., and Bell, J.F. 1992, Ap. J. Suppl. Ser, (in press).

Vassiliadis, E., and Wood, P.R. 1992, (in prep).

Wang, Qingde 1991, Mon. Not. R. Astr. Soc., 252, 47p.

Webster, B.L. 1975, M. N. R. A. S., 173, 437.

Webster, B.L. 1988, M.N.R.A.S., 230, 377.

Wood, P.R., Bessell, M.S., and Dopita, M.A. 1986, Ap. J., 311, 632.

Wood, P.R., and Faulkner, D.J. 1986, Ap. J., 307, 659.

Wood, P.R., Meatheringham, S.J., Dopita, M.A., and Morgan, D.H. 1987, Ap. J., 320, 178.

van den Bergh, S., 1991, IAU Symp.\#148, “The Magellanic Clouds", eds. R.Haynes and D. Milne, Kluwer:Dordrecht.

Zijlstra, A.A., 1990, Ast. Ap. 234, 387. 